\title{
MODIFICACIONES QUÍMICAS INDUCIDAS POR DOS ESPECIES DE LOMBRICES GEÓFAGAS EN SUELOS DE VERACRUZ, MÉXICO
}

\author{
Benito Hernández-Castellanos, ${ }^{1}$ Isabelle Barois, ${ }^{2}$ George G. Brown ${ }^{3}$ \& José \\ Antonio García-PÉREZ ${ }^{2}$ \\ ${ }^{1}$ Facultad de Biología, Universidad Veracruzana, Zona Universitaria, Circuito Presidentes, Xalapa, \\ Veracruz, México, benihernandez@uv.mx \\ ${ }^{2}$ Departamento Biología de Suelos, Instituto de Ecología, A.C. Ap. 63, Xalapa, Veracruz, México \\ ${ }^{3}$ Embrapa Florestas, Estrada da Ribeira, Km. 111, CP 319, 83411-000, Colombo-PR, Brasil
}

Hernández-Castellanos, B., I. Barois, G. G. Grown \& J. A. García-Pérez. 2010. Modificaciones químicas inducidas por dos especies de lombrices geófagas en suelos de Veracruz, México. Acta Zoológica Mexicana (n.s.), Número Especial 2: 295-308.

RESUMEN. Dos lombrices tropicales, una exótica (Pontoscolex corethrurus) y otra nativa (Glossoscolecidae sp.) de Veracruz, México, fueron cultivadas en condiciones de laboratorio para colectar sus respectivos turrículos (deyecciones) cada 48 horas, medir su tasa de producción y el efecto que ejercen sobre varios parámetros químicos en el suelo excretado. Ambas especies fueron cultivadas en un Lixisol (La Víbora) y P. corethrurus lo fue también en otros dos tipos de suelo, un Vertisol (Plan de las Hayas) y un Andosol (Los Tuxtlas). Los parámetros químicos medidos en los turrículos y en suelo testigo (control) fueron los siguientes: $\mathrm{pH}, \mathrm{C}, \mathrm{N}, \mathrm{C} / \mathrm{N}, \mathrm{NO}_{3}, \mathrm{NH}_{4}, \mathrm{Na}, \mathrm{K}, \mathrm{Ca}, \mathrm{Mg}, \mathrm{CIC}$, P-asimilable, P-Total, P-Resinas, Al, Fe, Cu, Zn, Mn. Para P. corethrurus la producción de turrículos superficiales fue más alta en los suelos con menor contenido de MO. En el Lixisol, Glossoscolecidae sp. produjo menor cantidad de turrículos comparada con $P$. corethrurus, pero promovió una disponibilidad mayor, especialmente de $\mathrm{K}, \mathrm{Na} \mathrm{Mg}, \mathrm{Mn}, \mathrm{Fe}, \mathrm{K}_{\text {y NH}}$. Pontoscolex corethrurus promovió también la concentración de la mayor parte de los parámetros estudiados con respecto al suelo control, tanto en el Andosol como en el Vertisol; en el Lixisol, esta especie incrementó la concentración en la mitad de los parámetros. Pontoscolex corethrurus tiene una capacidad alta de adaptarse a diferentes suelos y de modificar sus características químicas, promoviendo la disponibilidad de diversos elementos esenciales para el crecimiento vegetal; eso es especialmente importante en los suelos más pobres como el Lixisol.

Palabras clave: Mineralización, nutrientes, Pontoscolex corethrurus, Glossoscolecidae, especies exóticas y nativas

Hernández-Castellanos, B., I. Barois, G. G. Grown \& J. A. García-Pérez. 2010. Chemical changes induced by two geophagous earthworm species in soils from Veracruz, Mexico. Acta Zoológica Mexicana (n.s.), Número Especial 2: 295-308.

ABSTRACT. Two earthworm species, one exotic (Pontoscolex corethrurus) and the other one native (Glossoscolecidae sp.) from Veracruz, Mexico, were cultivated under laboratory conditions, to collect

Recibido: 16/05/2008; aceptado: 08/01/2010. 
their castings every $48 \mathrm{~h}$, measure cast production rates and their effects on soil chemical conditions and the concentration of several plant-available nutrients. Both species were cultivated in an Alfisol (La Víbora) and $P$. corethrurus was also raised in two other soil types, a Vertisol (Plan de las Hayas) and an Andosol (Los Tuxtlas), to measure $\mathrm{pH}, \mathrm{C}, \mathrm{N}, \mathrm{C} / \mathrm{N}, \mathrm{NO}_{3}, \mathrm{NH}_{4}, \mathrm{Na}, \mathrm{K}, \mathrm{Ca}, \mathrm{Mg}, \mathrm{CIC}$, available- totaland resin- $\mathrm{P}, \mathrm{Al}, \mathrm{Fe}, \mathrm{Cu}, \mathrm{Zn}, \mathrm{Mn}$ in the casts and control soil. $P$. corethrurus surface cast production was highest in soils with low organic matter content. In the Alfisol, Glossoscolecidae sp. produced less casts than $P$. corethrurus, but promoted the availability of most plant nutrients, especially $\mathrm{K}, \mathrm{Na} \mathrm{Mg}, \mathrm{Mn}, \mathrm{Fe}$, $\mathrm{K}$ and $\mathrm{NH}_{4}$. Pontoscolex corethrurus promoted the concentration of most parameters compared with the control in the Andosol and Vertisol; in the Alfisol, about half the parameters were significantly affected. Pontoscolex corethrurus has a high capacity to adapt to different soils and modify their chemical characteristics, promoting the availability of several plant nutrients; this is especially important in organic matter-poor soils, such as the Alfisol.

Keywords: Mineralization, nutrients, Pontoscolex corethrurus, Glossoscolecidae, exotic and native species

\section{INTRODUCCIÓN}

$\mathrm{Al}$ ingerir grandes cantidades de suelo, las lombrices geófagas tienen un papel clave en la estructura del suelo, la mineralización y redistribución de la materia orgánica del suelo (MO) y la dinámica de los nutrientes (Lavelle 1988, Edwards \& Bohlen 1996). Esta actividad de las lombrices puede contribuir a la restauración, mantenimiento o mejora de las propiedades físicas y químicas del suelo (Lavelle et al. 1989). Por ejemplo, una población de la especie geófaga Pontoscolex corethrurus (Müller, 1857) puede ingerir hasta 400 toneladas de suelo/ha/año en un pastizal de Laguna Verde, México (Lavelle et al. 1983), lo que demuestra la escala del impacto de esta lombriz en el suelo.

Se sabe que la actividad humana afecta en gran medida a las poblaciones de lombrices de tierra; muchas especies locales endémicas desaparecen como consecuencia de la alteración de su hábitat y/o por competencia con especies exóticas (Hendrix et al. 2006). La especie exótica $P$. corethrurus presenta una gran plasticidad adaptativa y un amplio rango de tolerancia a diferentes condiciones ambientales, por lo que se distribuye ampliamente en la región tropical, sobre todo en áreas agrícolas y alteradas (Lavelle et al. 1987, González et al. 2006), pero también en ecosistemas naturales (Brown et al. 2006). Esta especie tiene un gran potencial para el manejo, al estimular la productividad de los cultivos agrícolas (Brown et al. 1999, 2007). Sin embargo, su presencia puede también influir negativamente sobre las especies locales endémicas (Lapied \& Lavelle 2003), aunque no se sabe mucho de su potencial competitivo (e.g. García 2003, Hendrix et al. 2006) y su efecto en la dinámica de nutrientes en suelos invadidos.

Por lo tanto, el presente trabajo tuvo dos objetivos: 1) evaluar el efecto de la especie $P$. corethrurus sobre las propiedades químicas de tres tipos de suelo del estado de Veracruz, México; 2) determinar, comparativamente, el efecto de una especie exó- 
tica ( $P$. corethrurus) y una especie nativa (Glossoscolecidae sp.) en los parámetros químicos de un suelo en el que coexisten ambas especies. Los suelos usados tenían diferentes niveles de fertilidad y $P$. corethrurus se encuentra abundantemente en los suelos de Los Tuxtlas (Brown et al. 2001) y Plan de las Hayas (alcanzando biomasas de hasta $35 \mathrm{~g} / \mathrm{m}^{2}$ en este último sitio; Lavelle et al. 1981), aunque en el suelo de La Víbora es poco abundante, predominando la Glossoscolecidae sp. (Brown et al. datos no publicados).

\section{MATERIAL Y MÉTODOS \\ Tipos de suelo y especies de lombrices de tierra}

Los suelos utilizados en los experimentos se colectaron en tres localidades diferentes del Estado de Veracruz, México: Plan de las Hayas (1945' N 96³1' W, $800 \mathrm{~m}$ de altitud), Los Tuxtlas ( $18^{\circ} 35^{\prime} \mathrm{N} 95^{\circ} 04^{\prime} \mathrm{W}, 180 \mathrm{~m}$ de altitud) y La Víbora (18 $50^{\circ} \mathrm{N}$ $96^{\circ} 07^{\prime} \mathrm{W}, 35 \mathrm{~m}$ de altitud). El estrato muestreado correspondió a los primeros 10 $\mathrm{cm}$ del suelo. Los suelos se transportaron en costales al laboratorio, donde se secaron al aire y se pasaron por un tamiz de $2 \mathrm{~mm}$. Los suelos se identificaron según la clasificación Taxonómica de FAO (1987) y la "Soil Taxonomy" de Estados Unidos (USDA 1995): el suelo de Los Tuxtlas se clasificó como un Andosol (Andept), el de Plan de las Hayas como un Vertisol y el de La Víbora como un Lixisol (Haplustalf) (Cuadro I).

Las lombrices se colectaron manualmente del suelo y la especie endogea $P$. corethrurus se obtuvo en los tres sitios mencionados anteriormente. Los especimenes de la otra especie, también geófaga y endogea perteneciente a la familia Glossoscolecidae, sólo estuvieron presentes y se colectaron en la localidad de La Víbora. Esta especie pertenece a un nuevo género que aún no ha sido descrito (Fragoso, comunicación personal).

\section{Cultivos en el laboratorio}

Para la obtención de las deyecciones o turrículos se establecieron unidades de cultivo experimentales con cada una de las especies de lombrices en los sustratos en los que se

Cuadro I. Clasificación de los suelos de Los Tuxtlas, Plan de las Hayas y La Víbora según la FAO (1987) y la USDA Soil Taxonomy (1995), junto con su textura.

\begin{tabular}{llcccl}
\hline \multicolumn{1}{c}{ Localidad } & $\begin{array}{c}\text { Clase de suelo FAO } \\
\text { (USDA) }\end{array}$ & Arena (\%) & Limo (\%) & Arcilla (\%) & Textura \\
\hline Plan de las Hayas & Vertisol (Vertisol) & 27 & 40 & 33 & Migajón Arcilloso \\
Los Tuxtlas & Andosol (Andept) & 20 & 38 & 42 & Arcilloso \\
La Víbora & Lixisol (Haplustalf) & 82 & 7 & 11 & Areno Migajoso \\
\hline
\end{tabular}


colectaron, estableciendo los siguientes tratamientos según la metodología de Lavelle (1975): 1) Vertisol de Plan de las Hayas + P. corethrurus, 2) Andosol de Los Tuxtlas $+P$. corethrurus, 3) Lixisol de La Víbora + P. corethrurus, 4) Lixisol de La Víbora + Glossoscolecidae sp. y 5) suelo control (sin lombrices) para cada localidad.

Cada unidad de cultivo consistió en una caja de plástico de $15 \times 15 \times 5 \mathrm{~cm}$, en donde se depositaron $300 \mathrm{~g}$ de suelo tamizado a $2 \mathrm{~mm}$, humedecido con agua hasta alcanzar el $100 \%$ de su capacidad de campo. Para las unidades con lombrices se introdujeron individuos juveniles y adultos (5 individuos/recipiente) cuya biomasa promedio por unidad de cultivo fue de $387 \pm 27 \mathrm{mg}$ para $P$. corethrurus y $309 \pm 18 \mathrm{mg}$ para Glossoscolecidae sp.; el número de réplicas por tratamiento fue de 5 para las unidades con lombrices y 2 para las unidades control. En total se prepararon 26 unidades de cultivo las cuales se incubaron en una cámara de crecimiento a $25^{\circ} \mathrm{C}$.

Los turrículos superficiales se colectaron cada 48 horas y se pesaron para medir la tasa de producción por biomasa de lombriz por día. Una parte de ellos se usó para medir su contenido de humedad y otra parte se congeló para mantener sus características químicas, en espera de tener una cantidad suficiente de muestra para la realización de los análisis químicos. Simultáneamente se colectó aproximadamente la misma cantidad de suelo control y se congeló, dándole el mismo procesamiento que a los turrículos. Cada 14 días, se cambió el suelo de las unidades de cultivo y se pesaron las lombrices. La tasa de producción de las deyecciones se midió hasta los 132 días pero los cultivos se mantuvieron hasta los 8 meses, momento en el que se obtuvo la cantidad de muestra necesaria de turrículos para efectuar los diferentes análisis químicos.

\section{Análisis químicos}

La humedad se determinó por gravimetría y con balanza de humedad. El pH se midió en extracto acuoso en proporción 1:2.5 (peso: volumen) (McLean 1982). El porcentaje de $\mathrm{MO}$ y de $\mathrm{C}$ total se determinaron con el método de Walkley \& Black modificado (1947). El N total se determinó mediante el procedimiento micro-Kjeldhal (Bremmer 1965) y el N mineral (amonio y nitrato) por colorimetría (Cataldo \& Haroon 1975). El P extractable se midió según el método para suelos ácidos de Bray-Kurtz no. 1 modificado (Etchevers 1984), el $\mathrm{P}$ asimilable por el método de resinas intercambiables (Anderson et al. 1993) y el P total por colorimetría, según el método de digestión húmeda (Allan 1971, tomado de Etchevers 1984). El Na y K extractables se analizaron por fotometría (Chapman 1965), el Ca y Mg extractables mediante absorción atómica (Chapman 1965) y la Capacidad de intercambio catiónico (CIC) por el método de acetato de amonio (Chapman 1965). Finalmente, el Fe, $\mathrm{Cu}, \mathrm{Zn}, \mathrm{Mn}$ se determinaron por absorción atómica con DTPA (Lindsay et al. 1978) y el Al intercambiable por titulación (Chapman 1965). Todos los parámetros se midieron por duplicado. 


\section{Análisis estadísticos}

Los datos químicos del suelo y de los turrículos se analizaron de manera descriptiva, utilizando un análisis de componentes principales (ACP) para resumir el comportamiento general de las muestras en función de las distintas variables analizadas. Las diferencias significativas entre los distintos grupos en el ACP se evaluaron con análisis de varianza sobre los primeros dos componentes principales usando el módulo de Modelos Lineales Generalizados (MLG) y se utilizó el test a posteriori de HSD Tukey para determinar las comparaciones múltiples entre los distintos grupos.

Las diferencias puntuales en cada variable química, entre el suelo control y los turrículos de las lombrices, se evaluaron en los datos originales utilizando también el módulo de MLG y se aplicó la Prueba de comparaciones múltiples de Fisher (LSD) para determinar diferencias específicas entre tratamientos. Todos los análisis se efectuaron en el Software Statistica 8.

\section{RESULTADOS}

A lo largo del experimento, la producción de turrículos superficiales de $P$. corethrurus varió en los diferentes suelos y estas fluctuaciones fueron más importantes en el Lixisol y el Vertisol. La producción promedio de turrículos fue siempre mayor en el Lixisol de La Víbora ( $0.9 \mathrm{~g}$ de suelo seco/g de lombriz/día), seguido del Vertisol de Plan de las Hayas ( $0.4 \mathrm{~g}$ de suelo seco/g de lombriz/día) y el Andosol de los Tuxtlas ( $0.12 \mathrm{~g}$ de suelo seco/g de lombriz/día), observándose diferencias entre los tratamientos. Se pudo apreciar que a menor cantidad de $\mathrm{C}$ presente en el suelo, mayor es la tasa de ingestión por parte de $P$. corethrurus; la correlación del porcentaje de carbono presente en los diferentes suelos con la tasa de producción promedio de turrículos fue negativa y significativa $(\mathrm{r}=-0.8586, \mathrm{p}<0.05)$.

En general, la producción de turrículos de las dos especies fue diferente a lo largo del tiempo; $P$. corethrurus tuvo generalmente una mayor producción de turrículos en el Lixisol que Glossoscolecidae sp. (Fig. 1). En el día 53 no hubo producción superficial de turrículos y esto se debió a un enfriamiento brusco de la cámara de incubación.

A los 132 días de cultivo la biomasa de $P$. corethrurus se triplicó en el suelo de los Tuxtlas y se duplicó en el suelo de Plan de las Hayas. En cambio en el suelo de la Víbora la biomasa de $P$. corethrurus se mantuvo similar a la inicial y la de Glossoscolecidae sp. disminuyó casi el 50\%.

En el suelo de Los Tuxtlas (Andosol), la lombriz P. corethrurus promovió significativamente la concentración de nueve de los 19 parámetros estudiados, siendo mayor el incremento en el $\mathrm{Na}, \mathrm{NO}_{3}$ y C Total (Cuadro II). En el suelo de Plan de las Hayas (Vertisol) los turrículos de P. corethrurus presentaron un incremento en seis variables químicas (especialmente $\mathrm{NH}_{4}, \mathrm{Na}$ y $\mathrm{Cu}$ ) con respecto al testigo. Los otros 


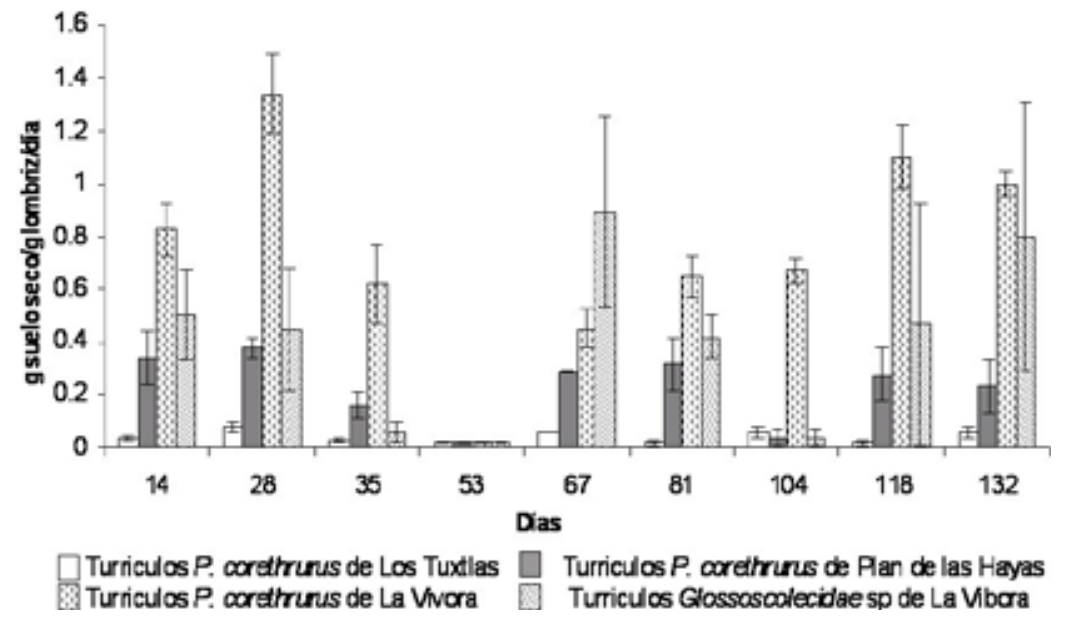

Figura 1. Producción de turrículos superficiales a lo largo del tiempo, por $P$. corethrurus en los suelos de Los Tuxtlas, Plan de las Hayas y la Víbora y por Glossoscolecidae sp. en el suelo de la Víbora (las barras indican el error estándar).

parámetros importantes como $\mathrm{C}$ Total y $\mathrm{pH}$ sólo presentan un incremento aproximado de $8 \%$.

En el suelo de La Víbora (Lixisol), P. corethrurus incrementó la concentración en ocho variables, principalmente $\mathrm{Mn}, \mathrm{NH}_{4}$ y C Total; en menor proporción, también afectó la relación $\mathrm{C} / \mathrm{N}$ y $\mathrm{P}$ extractable. En este suelo el efecto de selectividad de $\mathrm{MO}$ y mineralización por parte de $P$. corethrurus fue más evidente que en los otros suelos. Por su parte, Glossoscolecidae sp. promovió la mineralización de siete parámetros en este suelo, siendo su contribución más significativa para el $\mathrm{Mg}, \mathrm{Mn}, \mathrm{Fe}, \mathrm{K}, \mathrm{NH}_{4}$ (arriba del 50\%), C Total (43\%) y N Total (21\%) (Cuadro II). El incremento medio en la concentración de diversos elementos importantes para la nutrición vegetal para el caso de $P$. corethrurus fue mayor (22\%) en el suelo más pobre (La Víbora) que en el más rico (16\%, Los Tuxtlas) y el efecto de la especie de Glossoscolecidae fue aún mayor, con un incremento promedio de $43 \%$.

No se observó ninguna diferencia estadísticamente significativa en el P entre turrículos y los suelos testigo en los 4 tratamientos

El ACP logró resumir la variación en los datos y los dos primeros componentes explicaron el $88 \%$ de la variación total. El componente I representó la variación en $\mathrm{C}, \mathrm{N}, \mathrm{C} / \mathrm{N}, \mathrm{K}, \mathrm{Na}, \mathrm{Ca}, \mathrm{Mg}, \mathrm{CIC}, \mathrm{Fe}$ y Cu (Fig. 2A), y el componente II representó la variación en $\mathrm{pH}$ y Al, los cuales muestran una relación inversa. La visualización de los dos componentes (Fig. 2B) muestra claramente la formación de tres grupos que corresponden a los 3 suelos utilizados. Las deyecciones se diferenciaron claramente 


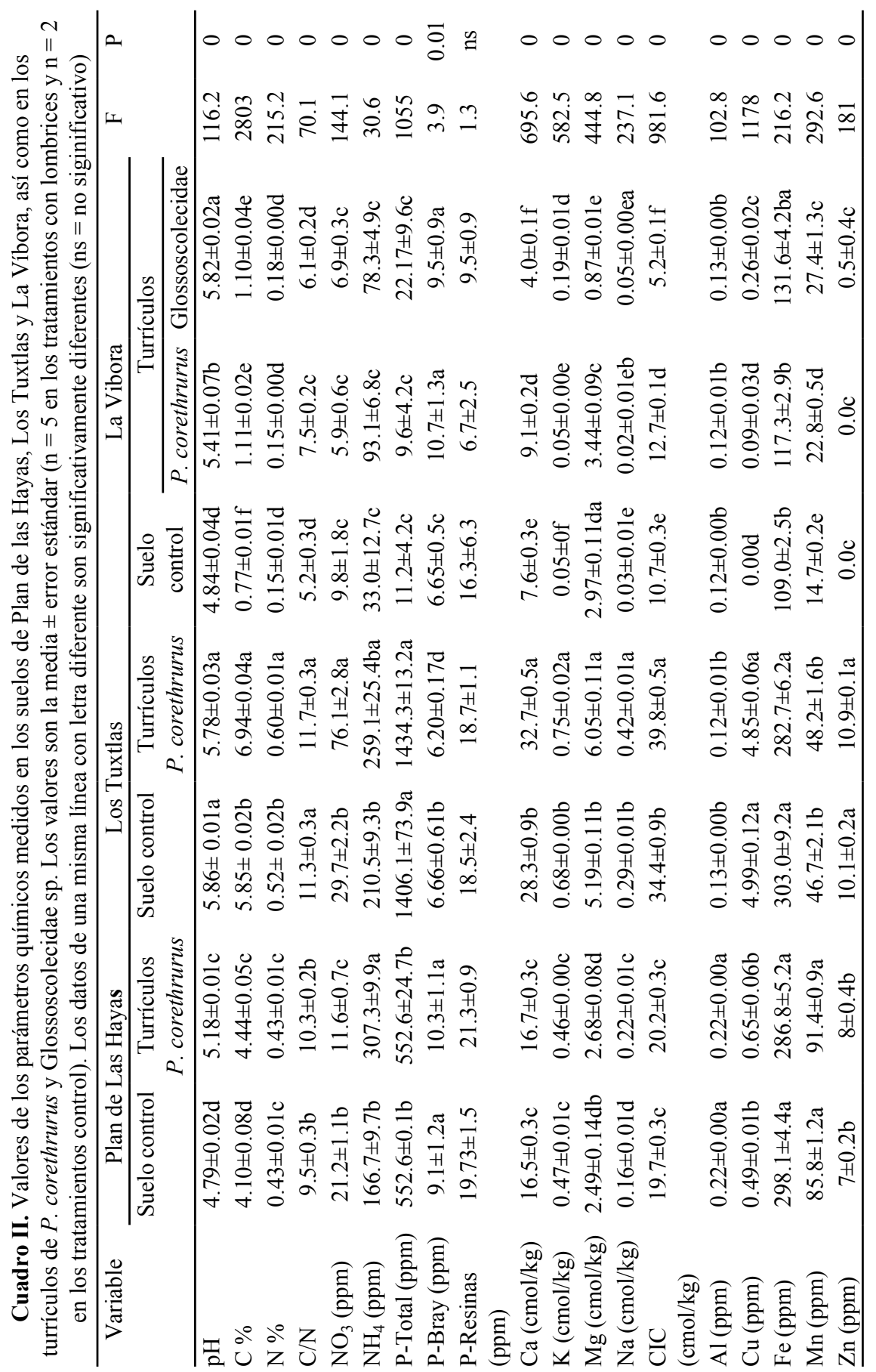




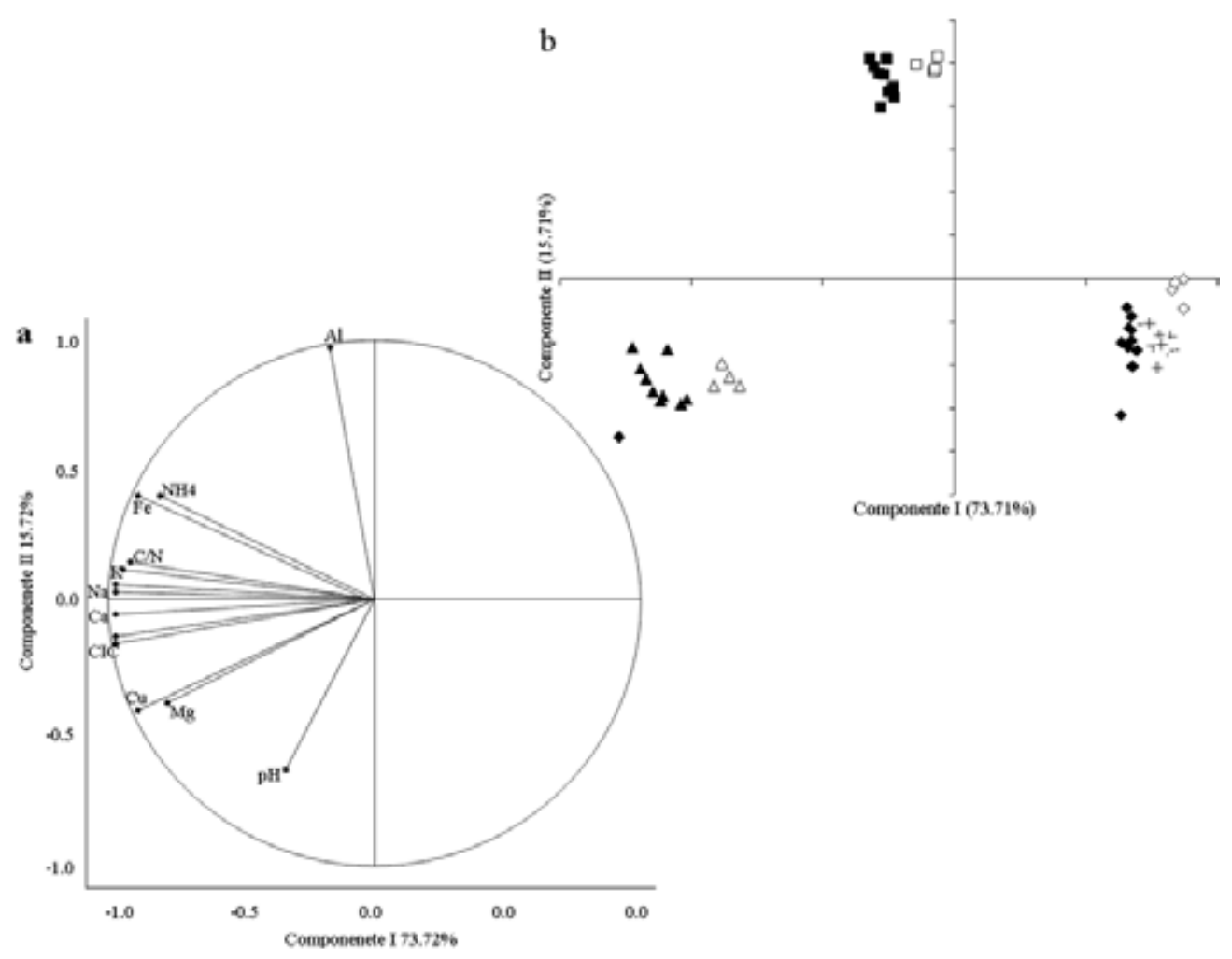

Figura 2. Resultado gráfico del Análisis de Componentes Principales (ACP) de los parámetros químicos de los suelos y de los turrículos de P. corethrurus y Glossoscolecidae sp. A: Circulo de correlación de los parámetros químicos con los dos primeros componentes principales que explican el $88 \%$ de la varianza de la variación total; el primer componente contribuye con el $73 \%$ y el segundo con el 15\%. B: proyección de los suelos y de los turrículos en el plano definido por el componente I y II. - : Turrículos de P. corethrurus y $\square$ : suelo control de Plan de las Hayas; $\boldsymbol{\Delta}$ : Turrículos de $P$. corethrurus y $\Delta$ : suelo control de Los Tuxtlas; $\bullet$ : Turrículos de P. corethrurus, +: Turrículos de Glossoscolecidae sp. y $\diamond$ : suelo control de La Víbora.

del suelo control al interior de cada una de las agrupaciones formadas, aunque estas diferencias son menos evidentes que entre los tipos de suelos. En todos los suelos donde se cultivó $P$. corethrurus, las variables responsables de las diferencias entre el suelo control y los turrículos fueron las representadas por el componente I $(\mathrm{C}, \mathrm{N}$, $\mathrm{C} / \mathrm{N}, \mathrm{K}, \mathrm{Na}, \mathrm{Ca}$ y CIC), mientras que para Glossoscolecidae sp., la agrupación estuvo determinada por las variables representadas en el componente II ( $\mathrm{pH}$ y Al).

El análisis de varianza efectuado sobre los componentes principales I y II, mostró diferencias significativas entre los suelos de cada sitio $(\mathrm{F}=1507, \mathrm{P}=0.00 ; \mathrm{y} \mathrm{F}=$ $724, \mathrm{P}=0.00$, respectivamente). La varianza explicada por el modelo para cada com- 
ponente, fue de $98.3 \%$ y de $96.5 \%$, respectivamente. Similarmente, la comparación univariada efectuada entre el suelo control y los turrículos fue significativa estadísticamente en prácticamente todas las variables (Cuadro II).

\section{DISCUSIÓN}

La mayor cantidad de suelo consumido por $P$. corethrurus correspondió al suelo de La Víbora, siendo éste el suelo con menor cantidad de MO. Esto confirma lo observado por Lavelle et al. (1989) y Barois et al. (1999) sobre la relación inversa entre el contenido de MO y la tasa de consumo de suelo por parte de las lombrices de tierra geófagas. Las tasas de producción de turrículos superficiales por parte de $P$. corethrurus fueron de 2 a 3 veces menores que las producciones totales (turrículos superficiales y dentro del suelo) observadas por Lavelle et al. (1987) y Senapati et al. (1993). Por tanto, se puede estimar que la producción de turrículos superficiales de $P$. corethrurus representó al menos un tercio de la producción total de turrículos bajo condiciones de laboratorio.

La producción de turrículos fue mayor para la especie exótica $P$. corethrurus en comparación con la endémica Glossoscolecidae sp. en el Lixisol de La Víbora. Por lo tanto, la especie nativa, de tamaño y peso similar a $P$. corethrurus, parece estar mejor adaptada a las condiciones de su suelo de origen en cuanto a su tasa de ingestión. Al estar en un suelo con poca MO y al estar menos adaptada a este suelo, $P$. corethrurus tiene que ingerir una mayor cantidad de suelo para satisfacer sus requerimientos, en comparación con Glossoscolecidae sp. Sin embargo, lombrices exóticas como P. corethrurus presentan gran plasticidad ecológica y son normalmente más eficaces que las lombrices endémicas para la utilización de recursos, lo cual les posibilita invadir nuevos hábitats (Lavelle et al. 1998, Trigo et al. 1999).

Diferentes autores han mencionado el efecto promotor que ejercen las lombrices en la mineralización y reciclaje de los nutrientes (Coq et al. 2007, Barot et al. 2007, Le Bayon \& Binet 2006, Blair et al. 1995, Barois et al. 1999, Bartz et al., este número). Los análisis químicos realizados a los turrículos y al suelo control indican que hay, de manera general, una mayor concentración de nutrientes en los turrículos (tanto de $P$. corethrurus como de Glossoscolecidae sp.); por consiguiente, las lombrices promovieron la mineralización y disponibilidad de los nutrientes en el suelo ingerido. Para el nitrógeno mineral, el efecto del pasaje por el intestino de las lombrices fue importante; $P$. corethrurus incrementó la concentración de $\mathrm{NH}_{4}$ en los tres suelos, llegando a más de $80 \%$ mayor en el Vertisol de Plan de las Hayas y en el Lixisol de La Víbora. Para el contenido de $\mathrm{NO}_{3}$ solo hubo un incremento significativo (de más de $150 \%$ ) en el Andosol de Los Tuxtlas. Diversos otros autores (por ejemplo, Tapia-Coral et al. 2006 y Barois et al. 1987) también observaron incrementos en el contenido de $\mathrm{NH}_{4}$ y $\mathrm{NO}_{3}$ en los turrículos de $P$. corethrurus. También hay que mencionar que 
en los suelos ácidos del presente experimento, las lombrices promovieron la neutralización del $\mathrm{pH}$ de los turrículos (especialmente en los suelos de Plan de las Hayas y La Víbora), lo cual puede afectar significativamente la disponibilidad de diversos elementos químicos (Lavelle et al. 1995).

El ACP mostró una distinción global clara en las propiedades químicas de los 3 suelos y, aunque en menor medida, también mostró una mayor concentración de nutrientes en los turrículos con respecto al suelo testigo. Los nutrientes que más cambiaron en valor en los turrículos con respecto al suelo control fueron el $\mathrm{C}, \mathrm{N}, \mathrm{NH}_{4}$, $\mathrm{Mn}, \mathrm{Na}, \mathrm{Ca}, \mathrm{Mg}, \mathrm{y} \mathrm{pH}$, y esto podría deberse a varias razones:

1) La contribución fisiológica de las lombrices, especialmente el incremento en Ca por acción de las glándulas calcíferas que liberan $\mathrm{CaCO}_{3}$ al intestino y que están presentes en ambas especies de lombrices. Esta secreción aumenta el Ca disponible en el intestino y el $\mathrm{pH}$ de los turrículos, el cuál afecta la forma y disponibilidad de otros nutrientes solubles para las plantas (Blair et al. 1995, Lal 1988, Mulongoy \& Bedoret 1989, Bartz et al., este número). Los resultados del presente trabajo confirman lo observado para $P$. corethrurus por Kale \& Krishnamoorthy (1980) y Bartz et al. (este número). Para la especie Glossoscolecidae sp., a pesar de su menor contribución al Ca de los turrículos, hubo un aumento en el pH de los mismos en comparación con el suelo testigo.

2) Una mezcla de efectos directos e indirectos de las lombrices. Esto ocurre, por ejemplo, con el $\mathrm{N}$ mineral. Una parte del aumento de $\mathrm{NH}_{4}$ en los turrículos es debido a la excreción de los nefridios, mientras otra se origina de la actividad microbiana intensa en el intestino de las lombrices, cuya comunidad incluye a los fijadores de $\mathrm{N}_{2}$ de vida libre y a los denitrificadores (Barois et al. 1987, Horn et al. 2003).

3) Selección de partículas orgánicas y del suelo ingerido por las lombrices. Dependiendo de la dieta, las lombrices ingieren mayor o menor cantidad de MO en diferentes estadios de humificación, así como partículas minerales de tamaños diferentes (Barois et al. 1993, 1999). Cuando la textura es más arenosa, el impacto de la lombriz en los nutrientes es más importante que en suelos con una textura mas fina, pues la lombriz selecciona partículas más delgadas (más arcillosas). En el presente trabajo, el efecto de P. corethrurus fue mayor en el Lixisol de La Víbora (textura gruesa) que el Andosol de Los Tuxtlas (textura fina). Esta selección puede llevar a aumentos en la disponibilidad de muchos nutrientes asociados a las placas de arcilla o a la MO del suelo como, por ejemplo, el P. En ese sentido, Kuczak et al. (2006) encontró en un Oxisol de Brasil aportes significativos de fósforo en turrículos inducidos por la ingestión y deyección de lombrices de la familia Glossoscolecidae. 
Los análisis estadísticos mostraron que el impacto de la especie nativa de Glossoscolecidae y la exótica $P$. corethrurus en el suelo de la Víbora es diferente. La especie nativa tiene un impacto más importante en la mineralización y la dinámica de los nutrientes. Hasta ahora no se puede decir que P. corethrurus esté acelerando los flujos biogeoquímicos en el suelo de la Víbora como González et al. (2006) lo mencionan para otros sitios. En cambio Lachnicht et al. (2002) mostraron que $P$. corethrurus inducía una reducción significativa de las tasas de mineralización de $\mathrm{C}$ y $\mathrm{N}$ cuando fue cultivada en mesocosmos junto con la nativa Estherella sp. en comparación a cuando fue cultivada individualmente. Futuros trabajos cultivando a las dos especies de este experimento juntas podrán inferir mejor en las posibles interacciones (positivas, neutras o negativas) entre ambas y el efecto sobre el ciclaje de nutrientes en el suelo de La Víbora.

En conclusión, los resultados del presente trabajo indican que en los tres tipos de suelo $P$. corethrurus seleccionó la MO y tuvo un efecto considerable en su mineralización y en la disponibilidad de nutrientes, siendo este más significativo en el Lixisol de La Víbora, el suelo más pobre y arenoso. Por otro lado la especie nativa de Glossoscolecidae parece estar mejor adaptada que la exótica $P$. corethrurus al suelo de La Víbora, pues ingirió menor cantidad de suelo y tuvo un mayor efecto sobre los contenidos de nutrientes que $P$. corethrurus. Sin embargo, la biomasa de Glossoscolecidae sp. disminuyó después de 132 días de cultivo a diferencia de la de $P$. corethrurus que aumentó hasta 3 veces, por lo que estas condiciones de cultivo para la especie nativa no fueron las optimas, y habría que hacer nuevas investigaciones para determinar cuales son sus requerimientos específicos, como de humedad o de aporte de MO.

\section{AGRADECIMIENTOS}

A Lourdes Cruz, Ninfa Portilla, Soraida Irissón y Martín de los Santos que apoyaron en la asesoría para la realización de los análisis químicos efectuados en el Laboratorio de Análisis Químicos de Suelos, Aguas y Plantas (LAQSAP) del Instituto de Ecología A.C., y en la elaboración de las figuras. Agradecemos también a los campesinos de cada localidad que nos permitieron muestrear en sus propiedades y a los revisores por sus comentarios valiosos. G. Brown agradece el apoyo del CNPq Brasil.

\section{LITERATURA CITADA}

Anderson, J. M. \& J. I. Ingram. 1993. Tropical soil biology and fertility. A handbook of methods. $2^{\text {nd }}$ Ed. CAB International, Oxford.

Barois, I., B. Verdier, P. Kaiser, A. Mariotti, P. Rangel, \& P. Lavelle. 1987. Influence of the tropical earthworm Pontoscolex corethrurus (Glossoscolecidae sp.) on the fixation and mineralization of Nitrogen. Pp. 151-158. In: A. M. Bonvicini-Pagliai \& P. Omodeo (Eds.). On earthworms. Mucchi Modena Editore, Bologna. 
Barois, I., G. Villemin, P. Lavelle \& F. Toutain. 1993. Transformation of the soil structure through Pontoscolex corethrurus (Oligochaeta) intestinal tract. Geoderma. 56: 57-66.

Barois, I., P. Lavelle, M. Brossard, J. Tondoh, M. Martínez, J.-P. Rossi, B. K. Senapati, A. Angeles, C. Fragoso, J. J. Jimenez, T. Decaëns, C. Lattaud, J. Kanyonyo, E. Blanchart, L. Chapuis, G. G. Brown \& A. G. Moreno. 1999. Ecology of earthworm species with large environmental tolerance and or extended distributions. Pp. 57-85. In: P. Lavelle, L. Brussaard \& P. F. Hendrix (Eds.). Earthworm management in tropical agroecosystems. CAB International, Wallingford.

Barot, S., A. Ugolini, \& F. B. Brikci. 2007. Nutrient cycling efficiency explains the long-term effect of ecosystem engineers on primary production. Functional Ecology. 21: 1-10.

Blair, J. M., R. W. Parmelee \& P. Lavelle. 1995. Influences of earthworms on biogeochemistry. Pp. 127-158. In: P. F. Hendrix (Ed.). Earthworm ecology and biogeography in North America. Lewis Publishers, Boca Raton.

Bouyoucos, G. J. A. 1951. Directions for making mechanical analysis of soil by the hydrometer. Soil Science. 42: 225-230.

Bray, R. H. \& L. T. Kurtz. 1945. Determination of P total organic and available phosphorus in soil. Soil Science. 59: 39-45.

Bremmer, J. M. 1965. Total nitrogen. Pp. 1149-1178. In: C.A. Black (Ed.). Methods of soil analysis, Part. 2. American Society of Agronomy, Madison.

Brown, G. G., B. Pashanasi, C. Gilot, J. C. Patrón, B. K. Senapati, S. Giri, I. Barois, P. Lavelle, R. J. Blakemore, A. V. Spain \& J. Boyer. 1999. Effects of earthworms on plant production in the tropics. Pp. 87-148. In: P. Lavelle, L. Brussaard \& P. F. Hendrix (Eds.). Earthworm management in tropical agroecosystems. CAB International, Wallingford.

Brown, G. G., I. Barois, \& P. Lavelle. 2000. Regulation of soil organic matter dynamics and microbial activity in the drilosphere and the role of interactions with other edaphic functional domains. European Journal of Soil Biology. 36: 1-23.

Brown, G. G., C. Fragoso, I. Barois, P. Rojas, J. C. Patrón, J. Bueno, A. G. Moreno, P. Lavelle, V. Ordaz \& C. Rodríguez. Diversidad y rol funcional de la macrofauna edáfica en los ecosistemas tropicales mexicanos. Acta Zoologica Mexicana (n.s.), No. especial. 1: 79-110.

Brown, G. G., S. W. James, A. Pasini, D. H. Nunes, N. P. Benito, P. T. Martins, K. D. Sautter. 2006. Exotic, peregrine and invasive earthworms in Brazil: diversity, distribution and effects on soils and plants. Caribbean Journal of Science. 42: 331-338.

Brown, G. G., B. K. Senapati, B. Pashanasi, C. Villenave, J. C. Patrón, P. Lavelle., I. Barois \& R. J. Blakemore. 2007. Earthworms stimulate plant production. Pp. 509-517. In: G. G. Brown \& C. Fragoso (Eds.). Minhocas na América Latina: biodiversidade e ecologia. Embrapa Soja, Londrina.

Cataldo, D. A., M. Haroon, L. E. Schrader \& V. L. Youngs. 1975. Rapid colorimetric determination of nitrate in plant tissue by nitration of salicylic acid. Communications in Soil Science and Plant Analysis. 6: 71-80.

Chapman, H. D. 1965. Cation Exchange capacity. Pp. 891-901. In: C. A. Black (Ed). Methods of soil analysis, Part 2. American Society of Agronomy, Madison.

Coq, S., B. G. Barthes. \& R. Oliver. 2007. Earthworm activity affects soil aggregation and organic matter dynamics according to the quality and localization of crop residues - An experimental study (Madagascar). Soil Biology \& Biochemistry. 39: 2119-2128.

Edwards, C. A. \& P. J. Bohlen. 1996. Biology and ecology of earthworms, $3^{\text {rd }}$ Ed. Chapman and Hall, London.

Etchevers, J. 1984. Técnicas de análisis químicos de suelos y plantas. Centro de Edafología, Colegio de posgraduados, Chapingo. 
FAO - UNESCO. 1987. Soil map of the world. Revised legend. World Resources Report 60. FAO, Rome.

García-Pérez, J. A. 2003. El potencial de las lombrices de tierra en la rehabilitación de suelos degradados por la industria del cemento: ensayos de laboratorio. Tesis de Doctorado en Ecología y Manejo de Recursos Naturales, Instituto de Ecología, A.C., Xalapa, México.

González, G., Y. Huang, X. Zou, \& C. Rodríguez. 2006. Earthworm invasions in the tropics. Biological Invasions. 8(6): 1247-1256.

Hendrix, P. F., G. H. Baker, M. A. Callaham, G. A. Damoff, C. Fragoso, G. González, S. W. James, S. L. Lachnicht, T. Winsome \& X. Zou. 2006. Invasion of exotic earthworms into ecosystems inhabited by native earthworms. Biological Invasions. 8(6): 1287-1300.

Horn, M. A., A. Schramm \& H. L. Drake. 2003. The earthworm gut: an ideal habitat for ingested $\mathrm{N}_{2} \mathrm{O}-$ producing microorganisms. Applied and Environmental Microbiology. 69(3): 1662-1669.

Kale, R.D. \& R.V. Krishnamoorthy. 1980. The calcium content of body tissues and castings of the earthworm Pontoscolex corethrurus (Annelida, Oligochaeta). Pedobiologia. 20: 309-315.

Kuczak, C. N., E. C. M. Fernandes, J. Lehmann, M. A. Rondon, \& F. J. Luizão. 2006. Inorganic and organic phosphorus pools in earthworm casts (Glossoscolecidae sp.) and a Brazilian rainforest oxisol. Soil Biology \& Biochemistry. 38: 553-560.

Lachnicht, S. W., P. F. Hendrix \& X. Zou. 2002. Interactive effects of native and exotics earthworms on resource use and nuturient mineralization in a tropical wet forest soil of Puerto Rico. Biology and Fertility of Soils. 36: 43-52.

Lal, R. 1988. Effects of macrofauna on soil properties in tropical ecosystems. Agriculture, Ecosystems and Environment. 24: 101-116.

Lapied, E. \& P. Lavelle. 2003. The peregrine earthworm Pontoscolex corethrurus in the East coast of Costa Rica. Pedobiologia. 47: 471-474.

Lavelle, P. 1975. Consommation annuelle d'une population naturelle de vers de terre (Millsonia anomala, Omodeo, Acanthodrilidae, Oligochaeta) dans la savane de Lamto (Côte d'ivoire). Revue d'Écologie et de Biologie du Sol. 12(1): 11-24.

Lavelle, P. 1988. Earthworm activities and the soil system. Biology and Fertility of Soils. 6: 237-251.

Lavelle, P., M. E. Maury \& V. Serrano. 1981. Estudio cuantitativo de la fauna del suelo en la región de Laguna Verde, Veracruz: Época de lluvias. Instituto de Ecología de México Publicaciones. 6: 75-105.

Lavelle, P., P. Rangel \& J. Kanyonyo. 1983. Intestinal mucus production by two species of tropical earthworms: Millsonia lamtoiana (Megascolecidae) and Pontoscolex corethrurus (Glossoscolecidae). Pp. 405-410. In: P. Lebrun, H. M. André, A. de Medts, C. Gregóire-Wibo \& G. Wauthy (Eds.). New trends in soil biology. Dieu-Brichart, Ottignies-Louvain-la-Neuve.

Lavelle, P., I. Barois, I. Cruz, C. Fragoso, A. Hernández, A. Pineda \& P. Rangel. 1987. Adaptative strategies of $P$. corethrurus (Glossoscolecidae, Oligochaeta), a peregrine geophagous earthworm of the humid tropics. Biology and Fertility of Soils. 5: 188-194.

Lavelle, P., I. Barois, A. Martin, Z. Zaidi \& R. Schaefer 1989. Management of earthworm populations in agro-ecosystems: A possible way to maintain soil quality. Pp. 109-122. In: M. Clarholm \& L. Bergstrom. (Eds.). Ecology of arable land. Kluwer Academic, Amsterdam.

Lavelle, P., A. Chauvel \& C. Fragoso. 1995. Faunal activity in acid soils. Pp. 201-211. In: R. A. Date, N. J. Grundon, G. E. Rayment \& M. E. Probert. (Eds). Plant-Soil Interactions at Low pH. Kluwer Academic Publishers, Amsterdam.

Lavelle, P., I. Barois., E. Blanchart, G. Brown., L. Braussaard, T. Decaens, C. Fragoso, J. Jiménez, K. Kajondo, M. Martínez, A. Moreno, B. Pashanasi, B. Senapati \& C. Villenave. 1998. Las 
lombrices como recurso en los agroecosistemas tropicales. Revista Naturaleza y Recursos. 1(34): 28-44.

Le Bayon, R. C. \& F. Binet 2006. Earthworms change the distribution and availability of phosphorous in organic substrates. Soil Biology \& Biochemistry. 38: 235-246.

Lindsay, W. L. \& W. A. Norvell. 1978. Development of a DTPA soil test for zinc, iron, manganese, and copper. Soil Science Society of America Journal. 42: 421-428.

McLean, E.O. 1982. Soil pH and lime requeriments. In: Pp. 199-224. In: A. L. Page, R. H. Miller \& D. R. Keeney (Eds.). Methods of soil analysis, Part 2. American Society of Agronomy, Madison.

Mulongoy, K. \& A. Bedoret. 1989. Properties of worm cast and surface soils under various plant covers in the humid tropics. Soil Biology \& Biochemistry. 21: 197-203.

Senapati, B. K., J. Sahu, B. Beheara \& P. S. Ramakrishnan. 1995. Impact of cropping and fallow period on ecological category of tropical earthworms and other macrofaunal components in shifting agriculture (Jhum) in East India. Pp. 119-130. In: Proceedings of the International Symposium on Integrated Exploitation and Sustainable Development in Red Soil Area. Nanachag, China.

Tapia-Coral, S. C., F. J. Luizão, E. Barros, B. Pashanasi \& D. del Castillo. 2006. Effect of Pontoscolex corethrurus (Müller, 1857) (Oligochaeta: Glossoscolecidae sp) inoculation on litter weight loss and soil nitrogen in mesocosms in the Peruvian Amazon. Caribbean Journal of Science. 42: 410-418.

Trigo, D., I. Barois, M. Garvín, E. Huerta, S. Irisson \& P. Lavelle. 1999. Mutualism between earthworms and soil microflora. Pedobiologia. 43: 866-873.

USDA Soil Survey Staff. 1995. Claves para la taxonomía de suelos. Sociedad Mexicana de la Ciencia del Suelo, México, D.F.

Walkley, A. L. \& A. Black. 1947. A rapid determination of soil organic matter. Journal of Agricultural Science. 25: 563-568. 